\title{
Regular Review
}

\section{Surgery for pancreatic cancer}

\author{
DAVID C CARTER
}

Pancreatic cancer presents an increasing diagnostic and therapeutic challenge. Over the past 40 years its incidence appears to have trebled in the United States ${ }^{1}$ and doubled in England and Wales. ${ }^{2}$ About 10 new cases now develop per 100000 population per year in most Western societies, and pancreatic cancer now ranks fifth and sixth in men and women respectively in the United States as a cause of death from cancer. $^{3}$

Despite the accuracy of new diagnostic techniques such as ultrasound, endoscopic retrograde cholangiopancreatography (ERCP), CT scanning, percutaneous transhepatic cholangiography, and angiography, diagnosis is usually still delayed until the disease has spread beyond the reach of surgical resection. The identification of "tumour markers" such as pancreatic oncofetal antigen ${ }^{4}$ offers promise for population screening and early diagnosis, but this promise remains far from clinical application.

At present, radical surgery offers the only prospect of cure, and the prognosis remains appalling. Most patients are dead within six months of diagnosis, some $90 \%$ are dead within a year, and survival beyond five years is exceptional-a gloomy background against which to review current surgical attitudes to the disease.

Limitations of surgical statistics-Surgeons have been reluctant to biopsy the pancreas, and inspection and palpation of the gland at laparotomy are notoriously inaccurate, leading to diagnostic error in as many as $25 \%$ of cases. ${ }^{6}$ Gudjonsson et $a l^{7}$ have suggested that histological confirmation is lacking in $40-60 \%$ of patients labelled as having pancreatic cancer. To collect 100 patients from their own Yale-New Haven hospital with histologically proved disease and adequate localisation of tumour they had first to review the records of 197 patients with a discharge diagnosis of pancreatic cancer. In 70 patients histological proof of the diagnosis was lacking, while in 27 cases cancer had arisen from the bile duct, duodenum, ampullary region, ovary, or an unknown location. The pancreas had been biopsied in only $13 \%$ of cases, the surgeons preferring to seek histological proof by biopsy of the liver or lymph nodes.

Comparison of the results achieved by different centres is complicated by the use of different denominators when determining survival rates. Some authors use all patients with pancreatic cancer while others count only those coming to surgery or consider only that subgroup undergoing radical operation. On the basis of 61 reported series Gudjonsson et al calculated that, on average, $78 \%$ of all patients with pancreatic cancer came to operation (range $46.5-97 \%$ ), and that $11 \%$ underwent resection (range $0-26 \%$ ). Using these figures to recalculate published survival statistics they suggested an absolute five-year survival rate of $0.4 \%$. Of 65 five-year survivors, eight had not undergone any form of resection: some authors claiming the most effective palliation by pancreatic resection appeared to have some of the highest mortality rates. ${ }^{7}$

Cancer arising in the periampullary region, distal common bile duct, or duodenum carries a more favourable prognosis than cancer arising from the pancreas. ${ }^{8}{ }^{9}$ Unfortunately, many authors still fail to distinguish clearly between these more favourable forms of cancer and cancer of the pancreas.

Confirmation of diagnosis-Histological confirmation of the diagnosis of pancreatic cancer is essential if statistics relating to the incidence of the disease are to become reliable, if the management of the individual is to become rational, and if unnecessary and potentially hazardous surgery is to be avoided. For example, at laparotomy it may prove impossible to distinguish between pancreatic cancer and chronic pancreatitis, and a gall stone impacted at the lower end of the common bile duct may readily masquerade as cancer. In one published series five of 88 patients undergoing pancreaticoduodenal resection were misdiagnosed as suffering from cancer, and one of these died postoperatively. ${ }^{10} \mathrm{~A}$ number of approaches are now available to avoid this problem.

Material for cytological examination may be obtained by collecting pure pancreatic juice after secretin stimulation or brushing the pancreatic duct at ERCP. Much depends on the skill of the endoscopist and cytologist, but the presence of cancer may be confirmed in some $54-79 \%$ of cases. ${ }^{11-13}$ DiMagno and $\mathrm{Go}^{14}$ imply that resectable tumours produce a lower yield of positive results than non-resectable tumours, but this is contrary to the experience of Mackie and associates, ${ }^{12}$ who suggest that tumours arising in the proximal pancreatic duct produce symptoms earlier and are more likely to exfoliate into a region accessible to a sampling cannula.

Percutaneous fine-needle aspiration of lesions localised radiologically may also be used to obtain material for cytological examination, ${ }^{15}$ giving a positive cytological diagnosis in $80 \%$ of patients with cancer in or around the pancreas. ${ }^{16}$ While spread of the tumour along needle tracks may be a hazard after biopsy with large-bore needles, tumour seeding has not been reported with needles finer than 21 gauge. ${ }^{16}$

Laparoscopy may be used to confirm the diagnosis of pancreatic cancer, assess spread, and avoid unnecessary laparotomy in some patients. ${ }^{17}$

The hazards attributed to open wedge biopsy of the pancreas have included bleeding, pancreatitis, and pancreatic fistula, and surgeons have been reluctant to biopsy the gland at laparotomy. Nevertheless, George and her colleagues ${ }^{18}$ confirmed the presence of pancreatic cancer by open wedge biopsy in 31 of their 35 patients without apparent complications or death. Other recent reports suggest a diagnostic yield ${ }^{16}$ 
of about $87 \%$ in cancer patients, with no mortality, and a complication rate of only $2^{\circ}{ }_{0}^{\prime}$.

Needle biopsy of the pancreas at laparotomy was first introduced to minimise the hazards attributed to open biopsy, but use of relatively wide-bore needles such as the VimSilverman gave ${ }^{16}$ a lower rate of confirmation of diagnosis $(72 \%)$, appreciable mortality $\left(2 \cdot 5^{\circ} \%\right)$, and a higher complication rate $(9 \cdot 3 \%)$. Intraoperative fine-needle aspiration biopsy now appears to be the method of choice if the diagnosis has not been confirmed before operation. The needle used has a cross-sectional area five times less than the Menghini and 14 times less than the Vim-Silverman needle.

Operation for pancreatic cancer-Since the first reports of successful pancreaticoduodenectomy, ${ }^{19}$ surgical opinion has been divided about the role of resection in treating cancer of the pancreas. Many surgeons argue that the long-term results of Whipple resection (partial pancreatectomy and duodenectomy) are too poor to justify the high incidence of postoperative complications and overall operative mortality of some $20 \% .^{7820}$ Such surgeons prefer palliative bypass of biliary obstruction, while some recommend duodenal bypass at the same time to avoid subsequent duodenal obstruction. ${ }^{9}$ Other surgeons argue that resection offers the only prospect of cure and that specialist centres can reduce current operative mortality and complication rates. Moossa and his group ${ }^{21}$ have carried out resection in $36 \%$ of their patients with pancreatic cancer over the past four years in Chicago, while Fortner and his group ${ }^{22}$ in New York undertook resection in $40 \%$ of a series of 52 patients presenting with cancer of the pancreas, ampulla, duodenum, or bile duct. Fortner mostly used regional pancreatectomy-consisting of total pancreatectomy, duodenectomy, en bloc dissection of regional lymph nodes, and excision of the pancreatic segment of portal vein. No fewer than 16 of 18 patients having the operation had been explored and their tumours deemed non-resectable at other centres. It remains to be seen whether such aggressive treatment will improve long-term survival results for patients with cancer of the pancreas, but its risks are reflected in an operative mortality rate of almost $17 \%$.

There have been no controlled trials comparing the results of palliative bypass with those of resection in the management of pancreatic cancer. Few surgeons would consider randomisation to be ethical or feasible. Shapiro ${ }^{20}$ made a reasoned attempt to compare the results of biliary bypass and Whipple resection by retrospective analysis of two groups of "good-risk" patients. Of 297 patients with a diagnosis of pancreatic cancer, 249 were excluded on the grounds that they did not undergo exploration or were found at laparotomy to have disseminated disease. The remaining 48 patients were divisible into 24 having Whipple resection and 24 undergoing bypass alone. All 48 were thought to have adenocarcinoma arising from ductal tissue within the head of the pancreas, but, whereas this was confirmed histologically in all patients having a resection, histological confirmation was lacking in twothirds of the bypass group. The two groups were comparable in terms of duration of symptoms and loss of weight, but the average age was five years lower in the resection group. Within these clearly stated limitations, the results of the study provide useful information. Bypass carried an operative mortality of $4 \%$ (one patient), whereas two treated by resection died in hospital at 65 and 69 days after surgery, an "in-hospital mortality" of $8 \%$. Patients treated by resection stayed in hospital for twice as long and the mean duration of their survival (10.6 months) did not differ significantly from that of patients treated by bypass ( $8 \cdot 1$ months). Life table analysis of cumulative survival rates showed no significant differences between the two groups, and to date there have been no fiveyear survivors.

On the basis of his own data and those of six similar retrospective reports, Shapiro ${ }^{20}$ concluded that patients with disease apparently limited to the pancreas treated by Whipple resection fared no better than those treated by bypass. Nevertheless, after a review of reports of 496 patients treated by Whipple resection, he pointed out that the 20 five-year survivors might have been denied their apparent cure had they merely undergone bypass surgery. Occasionally, however, patients survive five years after bypass. ${ }^{7}$ Furthermore, the mean operative mortality of Whipple resection in these patients was $21 \%$. As they were "good risk" patients, many of the 103 who died in the postoperative period might have been expected to survive for some six to 12 months had bypass been undertaken.

It is, indeed, the high operative mortality of Whipple resection that is crucial in any assessment of its place in treating pancreatic cancer. Much of the problem stems from the high complication rate which attends drainage of the pancreatic remnant into a loop of jejunum. Lower complication and mortality rates may possibly be achieved in specialist units; certainly Whipple resection should not be attempted by inexperienced surgeons. When a potentially resectable pancreatic cancer is found at laparotomy in a patient with obstructive jaundice the best course of action is insertion of a $\mathrm{T}$ tube high in the common bile duct and referral to a specialist centre. Even in the best hands the mortality will remain high, however; and many surgeons believe that Whipple resection should be abandoned for pancreatic cancer, and that they should practise palliative bypass surgery or opt for some safer form of resection.

Whipple operation or total pancreatectomy?-Total pancreatectomy removes the risk of postoperative pancreatitis and eliminates the need for pancreaticojejunal anastomosis and its attendant hazards, at the expense of removing all endocrine and exocrine pancreatic function. The operation of total pancreatectomy is not new, but the unsatisfactory results of the Whipple operation have prompted its reappraisal.

Comparison of the two operations is hampered by a lack of controlled data. It is made even more difficult by the inclusion of patients without cancer or those with cancer of the duodenum, ampullary region, or distal common bile duct. Pliam and ReMine $^{23}$ reported an operative mortality for total pancreatectomy of $21 \%$ in 1942-68, falling to $12.5 \%$ in $1969-73$, and to $7 \cdot 4 \%$ if only those operations performed since 1970 were considered. Nevertheless, the subgroup of patients undergoing total pancreatectomy for pancreatic cancer in 1969-73 had an operative mortality rate of $23 \%$. Ihse and colleagues $^{24}$ reported a hospital mortality of $23 \%$ in 65 patients undergoing total pancreatectomy over a 17-year period, 58 of whom had cancer of the pancreas. Even so, their mortality had fallen in recent years to $17 \%$ and was only $12 \%$ (six out of 48) when the operation was undertaken by senior surgeons trained in pancreatic surgery.

Overall, the mortality reported ${ }^{923-25}$ for total pancreatectomy for pancreatic cancer seems no better than that of Whipple resection-roughly $20 \%$. Possibly improved standards and techniques will reduce these rates to more acceptable levels, and many surgeons argue that the mortality after total pancreatectomy at least should not exceed that of Whipple resection.

Forrest and Longmire ${ }^{9}$ performed Whipple resection in 51 patients, all but one of whom had adenocarcinoma of the pancreas. On completion of the operation only 39 were 
thought to have had clearance of all obvious deposits of tumour, but after pathological examination of the resected specimen only 31 of these had resection lines clear of tumour. Survival rates were better in patients with clear resection lines, and patients undergoing "palliative" Whipple operations fared no better than those having bypass surgery. Forrest and Longmire suggested that total pancreatectomy offered a better prospect of excising all pancreatic tumour, an argument reinforced by Ihse and colleagues, ${ }^{24}$ who found tumour cells beyond the usual Whipple resection line in nine of 58 total pancreatectomy specimens. Multicentricity may also have to be considered as a source of residual pancreatic tumour after partial pancreatectomy.

Cubilla and colleagues ${ }^{26}$ carried out a detailed histological examination of resection specimens in 22 cases of pancreatic adenocarcinoma. Only three patients had no detectable metastases in lymph nodes; 10 had metastases in only one group of nodes (usually along the upper border of the head or body of the pancreas), and nine had metastases in more than one group of nodes. One-third of these patients had disease in lymph nodes not normally removed in the Whipple resection.

These findings support the case for radical surgery more extensive than Whipple resection; but pancreatic cancer may be analogous to breast cancer in that spread to the regional lymph nodes may denote distant metastasis. Nevertheless, there can be no justification for persevering with the Whipple operation if the penalties of carrying out an operation less radical than total pancreatectomy are not outweighed by a lower operative mortality.

Comparison of long-term survival after the various forms of pancreatic resection is impossible in the absence of controlled data. The root problem remains one of late diagnosis rather than extent of resection, and any improvement in five-year survival rates from widespread application of total pancreatectomy seems destined to be marginal.

Diabetes is inevitable after total pancreatectomy. Advocates of total pancreatectomy point out that some $16 \%$ to $38 \%$ of patients with pancreatic cancer are diabetic preoperatively, ${ }^{9} 23$ and that diabetes is not uncommon after Whipple resection. ${ }^{24} 25$ Patients who have had a total pancreatectomy are very sensitive to insulin, and half of them will have hypoglycaemic episodes while in hospital after operation. ${ }^{22}$ Postpancreatectomy diabetes is, however, rarely difficult to manage. ${ }^{9} 2325$ Once the patient has recovered from the immediate effects of surgery, however, both patient and general practitioner must be made aware of the potential danger of insulin sensitivity, and the ease with which fatal hypoglycaemia may be induced.

Exocrine insufficiency remains a major problem after both partial and total pancreatectomy, and steatorrhoea is the main disadvantage of pancreatic resection. In theory, worthwhile exocrine pancreatic function should be retained after Whipple resection, but the clinical results are often disappointing. Forrest and Longmire ${ }^{9}$ believe that the pancreatic ducts and pancreaticojejunal anastomosis may stenose after a variable interval. Examination of pancreatic resection specimens shows fibrosis and degeneration in the body and tail of the gland by the time of surgery in almost half of patients with carcinoma of the head of pancreas. ${ }^{24}$

Individual susceptibility to steatorrhoea varies greatly after total pancreatectomy. Oral pancreatic supplements should be prescribed routinely, and most patients eventually pass two to three stools a day after a much more frequent bowel habit in the early postoperative period. Nevertheless, steatorrhoea may prove incapacitating in some patients despite increased intake of enzyme preparations. Non-specific drugs may be useful for slowing intestinal transit in such individuals.

The loss of pancreatic bicarbonate secretion after pancreatectomy predisposes to peptic ulceration, and partial gastrectomy or truncal vagotomy with antrectomy are now essential extensions of pancreaticoduodenal resection. Further reduction in the secretion of acid and pepsin by histamine $\mathrm{H}_{2}$ receptor antagonists or antacids may be useful in patients with persistent troublesome steatorrhoea. ${ }^{27}$

The loss of endogenous pancreatic exocrine secretion is reflected in restriction of physical activity and failure to regain weight after operation. Patients rarely regain their ideal weight after pancreatectomy, but progressive loss of weight is often an indication of progression of malignant disease.

St Mungo professor of surgery

DAVID C CARTER

Glasgow University

Honorary consultant surgeon

Glasgow Royal Infirmary

${ }^{1}$ Krain LS. The rising incidence of carcinoma of the pancreas: real or apparent? F Surg Oncol 1970;2:115-24.

2 Office of Population Censuses and Surveys. Cancer mortality, England and Wales 1911-1970. Studies on medical and population subjects no 29. London: HMSO, 1975.

${ }^{3}$ Aoki K, Ogawa H. Cancer of the pancreas: international mortality trends. World Health Statistics Quarterly 1978;31:2-27.

4 Gelder F, Reese C, Moossa AR, Hunter R. Studies on an oncofetal antigen, POA. Cancer 1978;42:1635-45.

5 Banwo O, Versey J, Hobbs JR. New oncofetal antigen for human pancreas. Lancet $1974 ; \mathrm{i}: 643-5$.

${ }^{6}$ Fortner JG. Regional resection of cancer of the pancreas: a new surgical approach. Surgery 1973;73:307-20.

7 Gudjonsson B, Livstone EM, Spiro HM. Cancer of the pancreas. Diagnostic accuracy and survival statistics. Cancer 1978;42:2494-506.

8 Smith R. Cancer of the pancreas. F R Coll Surg Edinb 1978;23:133-50.

9 Forrest JF, Longmire WP. Carcinoma of the pancreas and periampullary region. A study of 279 patients. Ann Surg 1979;189:129-38.

10 Gilsdorf RB, Spanos P. Factors influencing morbidity and mortality in pancreaticoduodenectomy. Ann Surg 1973;177:332-7.

11 Hatfield ARW, Smithies A, Wilkins R, Levi AJ. Assessment of endoscopic retrograde cholangiopancreatography (ERCP) and pure pancreatic juice cytology in patients with pancreatic cancer. Gut 1976;17:14-21.

12 Mackie CR, Dhorajiwala J, Blackstone MO, Bowie J, Moossa AR. Value of new diagnostic aids in relation to the disease process in pancreatic cancer. Lancet 1979;ii:385-8, 389.

${ }^{13}$ Endo Y, Morii T, Tamura H, Okuda S. Cytodiagnosis of pancreatic malignant tumours by aspiration under direct vision, using a duodenal fiberscope. Gastroenterology 1974;67:944-51.

14 DiMagno EP, Go VLW. Cytodiagnosis of pancreatic cancer. Gastrointest Endosc $1977 ; 23: 173-4$.

15 Hancke S, Holm $\mathrm{HH}$, Koch F. Ultrasonically guided percutaneous fine needle biopsy of the pancreas. Surg Gynecol Obstet 1975;140:361-4.

${ }^{16}$ McLoughlin MJ, Ho CS, Langer B, McHattie J, Tao LC. Fine needle aspiration biopsy of malignant lesions in and around the pancreas. Cancer 1978;41:2413-9.

17 Cuschieri A, Hall AW, Clark J. Value of laparoscopy in the diagnosis and management of pancreatic carcinoma. Gut 1978;19:672-7.

18 George P, Brown C, Gilchrist J. Operative biopsy of the pancreas. $B r \mathcal{F}$ Surg 1975;62:280-3.

19 Whipple AO, Parsons WB, Mullins CR. Treatment of carcinoma of the ampulla of Vater. Ann Surg 1935;102:763-79.

20 Shapiro TM. Adenocarcinoma of the pancreas: a statistical analysis of biliary bypass vs Whipple resection in good risk patients. Ann Surg 1975;182:715-21.

${ }^{21}$ Mackie CR, Cooper MJ, Lewis MH, Moossa AR. Non-operative differentiation between pancreatic cancer and chronic pancreatitis. Ann Surg $1979 ; 189: 480-7$

22 Fortner JG, Kim DK, Cubilla A, Turnbull A, Pahnke LD, Shils ME. Regional pancreatectomy: en bloc pancreatic, portal vein and lymph node resection. Ann Surg 1977;186:42-50.

${ }^{23}$ Pliam MB, ReMine WH. Further evaluation of total pancreatectomy. Arch Surg 1975;110:506-11.

24 Ihse I, Lilja P, Arnesjö B, Bengmark S. Total pancreatectomy for cancer. An appraisal of 65 cases. Ann Surg 1977;186:675-80.

${ }^{25}$ Brooks JR, Culebras JM. Cancer of the pancreas. Palliative operation, Whipple procedure or total pancreatectomy? Am f Surg 1976;131: 516-9.

${ }^{26}$ Cubilla AL, Fortner J, Fitzgerald PJ. Lymph node involvement in carcinoma of the head of the pancreas area. Cancer 1978;41:880-7.

27 Saunders JHB, Drummond S, Wormsley KG. Inhibition of gastric secretion in treatment of pancreatic insufficiency. Br Medf $1977 ; \mathrm{i}: 418-9$. 